\title{
Postoperative adjuvant transarterial chemoembolization for multinodular hepatocellular carcinoma within the Barcelona Clinic Liver Cancer early stage and microvascular invasion
}

\author{
Han Wang ${ }^{1,2,3}$, Peng-Cheng $\mathrm{Du}^{4}$, Meng-Chao $\mathrm{Wu}^{5}$, Wen-Ming Cong ${ }^{1,2,3}$ \\ ${ }^{1}$ Department of Pathology, Eastern Hepatobiliary Surgery Hospital, The Second Military Medical University, Yangpu, Shanghai 200438, China; ${ }^{2}$ Key \\ Laboratory of Signaling Regulation and Targeting Therapy of Liver Cancer (Second Military Medical University) Ministry of Education, Yangpu, \\ Shanghai 200438, China; ${ }^{3}$ Shanghai Key Laboratory of Hepatobiliary Tumor Biology (Eastern Hepatobiliary Surgery Hospital), Yangpu, Shanghai \\ 200438, China; ${ }^{4}$ Department of Vascular Surgery, Changhai Hospital, ${ }^{5}$ Department of Surgery, Eastern Hepatobiliary Surgery Hospital, The Second \\ Military Medical University, Yangpu, Shanghai 200433, China \\ Contributions: (I) Conception and design: H Wang, WM Cong; (II) Administrative support: WM Cong; (III) Provision of study material or patients: \\ MC Wu; (IV) Collection and assembly of data: H Wang, PC Du; (V) Data analysis and interpretation: H Wang; (VI) Manuscript writing: All authors; \\ (VII) Final approval of manuscript: All authors. \\ Correspondence to: Wen-Ming Cong. Department of Pathology, Eastern Hepatobiliary Surgery Hospital, 225 Changhai Rd., Yangpu, Shanghai \\ 200438, China. Email: wmcong@smmu.edu.cn.
}

Background: The survival benefit of postoperative adjuvant transcatheter arterial chemoembolization (PA-TACE) remained controversial. We aimed to investigate the prognosis effect of PA-TACE on the Barcelona Clinic Liver Cancer (BCLC) early stage multinodular hepatocellular carcinoma (MHCC) patients with/without microvascular invasion (MVI).

Methods: Two hundred and seventy-one patients from January 2010 to December 2014 undergoing curative hepatectomy were included in this study. Disease-free survival (DFS) rates and overall survival (OS) rates as well as prognostic factors were analyzed by the Kaplan-Meier method, the log-rank test and the Cox proportional hazard model.

Results: Thirty-four point four percent (44/128) MVI positive and 55.2\% (79/143) MVI negative patients underwent PA-TACE. Multivariate analysis revealed that HBV DNA load $>10^{3}$ copy/mL, $>$ three tumors, MVI, and without PA-TACE were independent risk factors for poor DFS. Higher alkaline phosphatase (ALP), three tumors, MVI, and without PA-TACE were independent risk factors for poor OS. Both DFS and OS were significantly improved in patients with MVI who received PA-TACE as compared to those who underwent hepatic resection alone (5-year DFS, 26.3\% vs. 20.7\%, P=0.038; 5-year OS, 73.6\% vs. $47.7 \%, \mathrm{P}=0.005)$. No differences were noted in DFS and OS among MVI negative patients with or without PA-TACE (5-year DFS, 33.7\% vs. 33.0\%, P=0.471; 5-year OS, 84.1\% vs. 80.3\%, P=0.523). Early recurrence was more likely to occur in patients without PA-TACE $(\mathrm{P}=0.001)$.

Conclusions: PA-TACE was a safe intervention and could effectively prevent tumor recurrence and improve the survival of the BCLC early stage MHCC patients with MVI.

Keywords: Hepatocellular carcinoma (HCC); microvascular invasion (MVI); clonal origin; postoperative adjuvant transcatheter arterial chemoembolization (PA-TACE)

Submitted Jul 16, 2018. Accepted for publication Sep 25, 2018.

doi: $10.21037 / \mathrm{hbsn} .2018 .09 .05$

View this article at: http://dx.doi.org/10.21037/hbsn.2018.09.05 


\section{Introduction}

Liver cancer is the 4th most common malignancy and the 3 rd leading cause of cancer-related death in China (1). Hepatocellular carcinoma (HCC) is the most frequent pathological type of liver cancer (2). In the various clinical stages of HCC, the Barcelona Clinic Liver Cancer (BCLC) stage was the most widely used staging system in clinical practice (3). The BCLC stage recommended curative treatment methods for the HCC patients with single nodule or $\leq 3$ nodules with none $>3 \mathrm{~cm}$, Child-Pugh classification A-B, Eastern Cooperative Oncology Group (ECOG) $=0$, and without gross vascular invasion and extrahepatic metastasis, which was defined as the early stage (4). The 5 -year survival rate of these patients can reach $70-80 \%$ after hepatectomy (5-7).

With the deepen cognition of molecular mechanism of HCC, it was currently believed that there were at least two clonal origin patterns of multinodular HCC (MHCC), namely, the pattern of intrahepatic metastasis (IM type) and multicentric occurrence (MO type) (8). The IM type HCC was confirmed to have a higher postoperative recurrence rate and poorer survival prognosis than the MO type HCC $(9,10)$. Microvascular invasion (MVI) was demonstrated to be significantly correlated with the IM type MHCC (11). Therefore, considering the complex and expensive molecular pathological examination of clonal origin, MVI was potential as an indicator of the selection strategy of stratified therapy for the BCLC early stage MHCC with different clonal origin.

Transcatheter arterial chemoembolization (TACE) was the most general palliative treatment for unresectable MHCC (12). Effective TACE achieved satisfied results in eliminating tiny invisible tumor spots with little complication. Therefore, it was widely used in preoperative and postoperative adjuvant therapy $(13,14)$. HCC patients beyond the BCLC early stage has been illustrated to benefit from postoperative adjuvant TACE (PA-TACE) $(15,16)$. However, the choice of the BCLC early stage MHCC patients for PA-TACE remained unclear.

Therefore, our study aimed to investigate the efficacy of PA-TACE on the BCLC early stage MHCC patients. MVI was further explored to distinguish the efficacy of PA-TACE.

\section{Methods}

\section{Patients}

We retrospectively identified 271 HCC patients who received curative surgical resection between January 2010 and December 2014 at the Eastern Hepatobiliary Surgery Hospital. The BCLC early stage MHCC criteria was utilized as a broad framework for our study (3): (I) MHCC within $\leq 3$ nodules, none $>3 \mathrm{~cm}$ according to preoperative imaging findings, (II) Child-Pugh classification A or B, (III) $\mathrm{ECOG}=0$, (IV) having undergone curative resection. Exclusion criteria: (I) gross vascular invasion or extrahepatic metastasis, (II) previous history of treatment of malignancy, (III) perioperative mortality, (IV) intrahepatic recurrence within 1 month after surgery, which ensured that PA-TACE was impossible. The flow chart of the entire process was shown in Figure 1.

The 7-point baseline sampling protocol was performed for all the specimens based on the "Evidence-based Practice Guidelines for Standardized Pathological Diagnosis of Primary Liver Cancer in China: 2015 Update" (17). The diagnosis of HCC was made by two different pathologists based on histopathology (17). MVI was defined as the presence of tumor cells in a portal vein, hepatic vein, or large capsular vessel of the surrounding hepatic tissue lined by endothelium that was visible only on microscopy (18). The diameter of tumor was based on the sum of maximum diameter of every tumor. The capsule was depended on the maximum diameter of tumor. The Edmondson-Steiner grading (III-IV or I-II) was judged on the highest grade found in the specimen.

Patient identities were anonymized before analysis. The requirement for informed consent was waived. The study protocol was approved by the Eastern Hepatobiliary Surgery Hospital Research Ethics Committee.

\section{Treatments}

All patients were treated by curative hepatic resection, which was defined as no residual tumor and a negative resection margin based on the histological examination. After excluding patients who did not suitable for PA-TACE, all patients were recommended to undergo PA-TACE at 1-3 months after the operation when the liver function of the patients had recovered. Whether the patients followed the physician's recommendation mainly depended on their socioeconomic status and their compliance with the doctors.

During the PA-TACE, a hepatic arterial catheter was placed into the proper hepatic artery through the femoral artery using the Seldinger technique, and TACE was conducted for the entire remnant liver. Hepatic angiography, computed tomography (CT) angiography, 


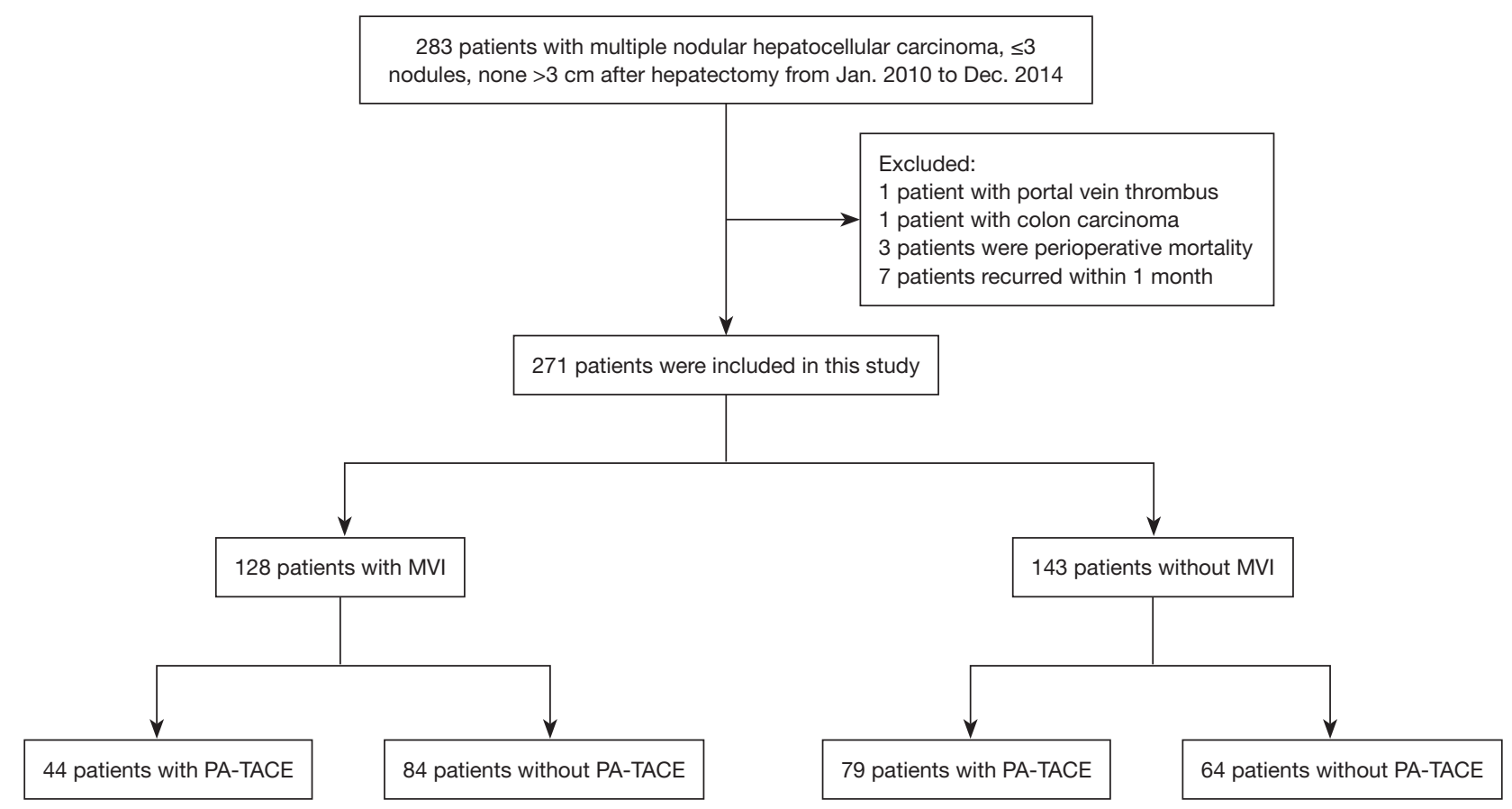

Figure 1 Flow chart of multinodular hepatocellular carcinoma patients within the Barcelona Clinic Liver Cancer early stage according to microvascular invasion and postoperative adjuvant transarterial chemoembolization.

or both were performed to detect any obvious tumor stains in the remnant liver. If no tumor stain was found, chemotherapeutic agents, lipiodol $(5-10 \mathrm{~mL})$, doxorubicin hydrochloride (10 mg), pirarubicin (THP), or pharmorubicin (20-40 mg) was injected through the microcatheter. The dosage of lipiodol and doxorubicin was determined by body surface area and underlying liver function (14).

Recurrence was defined as suspicious imaging findings or biopsy-proven tumor. When tumor recurrence was diagnosed, the therapeutic strategy was evaluated based on number of tumors, tumor location, liver function and general patient condition. Treatment with curative intent included surgical re-resection and ablation. Other treatment methods contained TACE, radiotherapy, chemotherapy, percutaneous ethanol injection therapy, sorafenib and Chinese traditional medicine.

\section{Follow-up}

The patients were followed up once every 2 months in the first 2 years after operation and then once every 3 months thereafter. Liver function assessments, tumor markers, and abdominal ultrasounds were performed at each follow-up visit. Contrast-enhanced CT or magnetic resonance imaging was performed once every 6 months or when recurrence/ metastasis was suspected. Disease-free survival (DFS) and overall survival (OS) were used as study endpoints. DFS was calculated from the date of surgery to the date when recurrence and/or metastasis. OS was defined as the duration between surgery and the last follow-up visit or HCC-related death. Data in this study were censored on April 30th, 2018.

\section{Statistical methods}

The continuous variables and the categorical variables are expressed as the mean \pm standard deviation and $\mathrm{n}(\%)$, respectively. The continuous variables were compared by the student's $t$-test or the Mann-Whitney $\mathrm{U}$ test. The categorical variables were evaluated using the $\chi^{2}$ test or the Fisher's exact test, as appropriate. The relative prognostic risk factors in predicting DFS and OS were assessed by the Cox proportional hazards regression models. The Kaplan-Meier curves and the log-rank test were used to analyze DFS and OS. A P value less than 0.05 was considered significant. The statistical analyses of the data were performed using SPSS 22.0 for Windows (IBM, New York, USA). 


\section{Results}

\section{Clinicopathological features}

According to the inclusion and exclusion criteria, 271 patients were entered into our study. The median follow-up time was 63.3 months (range, 3.87-98.97 months) for all patients. Based on the postoperative histopathological examination, 128 (47.2\%) patients and 143 (52.8\%) patients were MVI positive and MVI negative, respectively. Among the MVI positive patients, $44(34.4 \%)$ underwent PA-TACE; with MVI negative patients, of these 79 (55.2\%) underwent PA-TACE (Table 1). Patients with/without PA-TACE presented similar peri- or post-operative clinicopathological characteristics except the incidence of cirrhosis in the MVI positive cohort $(\mathrm{P}=0.008)$. The median interval between resection and PA-TACE was 46.5 days (range, 26-92 days). No patient had serious postoperative complications. Sixtyone (49.6\%), 7 (5.7\%), 5 (4.1\%), and 15 (12.2\%) patients

Table 1 Baseline clinicopathological characteristics of patients

\begin{tabular}{|c|c|c|c|c|c|c|}
\hline Characteristics & \multicolumn{3}{|c|}{ MVI negative $(n=143)$} & \multicolumn{3}{|c|}{ MVI positive $(n=128)$} \\
\hline Sex & & & 0.071 & & & 0.516 \\
\hline Male & $74(93.7 \%)$ & $54(84.4 \%)$ & & $42(95.5 \%)$ & $76(90.5 \%)$ & \\
\hline Female & $5(6.3 \%)$ & $10(15.6 \%)$ & & $2(4.5 \%)$ & $8(9.5 \%)$ & \\
\hline TBIL, $\mu \mathrm{mol} / \mathrm{L}$ & $14.37 \pm 4.95$ & $13.01 \pm 4.75$ & 0.058 & $13.53 \pm 4.74$ & $15.16 \pm 6.21$ & 0.193 \\
\hline $\mathrm{TP}, \mathrm{g} / \mathrm{L}$ & $69.90 \pm 6.31$ & $68.99 \pm 5.37$ & 0.362 & $70.75 \pm 6.17$ & $69.57 \pm 5.32$ & 0.262 \\
\hline ALB, g/L & $42.03 \pm 3.95$ & $41.33 \pm 3.62$ & 0.276 & $42.73 \pm 3.85$ & $42.19 \pm 3.48$ & 0.427 \\
\hline ALT, U/L & $40.45 \pm 24.67$ & $38.30 \pm 22.59$ & 0.630 & $37.99 \pm 14.01$ & $40.95 \pm 22.49$ & 0.954 \\
\hline WBC, $10^{9} / \mathrm{L}$ & $5.29 \pm 1.39$ & $4.97 \pm 1.39$ & 0.180 & $5.17 \pm 1.36$ & $4.97 \pm 1.35$ & 0.415 \\
\hline $\mathrm{RBC}, 10^{\circ} / \mathrm{L}$ & $4.66 \pm 0.49$ & $4.53 \pm 0.42$ & 0.091 & $4.66 \pm 0.46$ & $4.57 \pm 0.56$ & 0.243 \\
\hline $\mathrm{PLT}, 10^{\circ} / \mathrm{L}$ & $139.70 \pm 49.54$ & $140.02 \pm 56.41$ & 0.733 & $150.89 \pm 43.93$ & $134.38 \pm 48.55$ & 0.062 \\
\hline INR & $1.00 \pm 0.07$ & $1.00 \pm 0.07$ & 0.959 & $0.99 \pm 0.06$ & $1.01 \pm 0.09$ & 0.298 \\
\hline $\mathrm{PT}, \mathrm{s}$ & $12.05 \pm 0.81$ & $12.02 \pm 0.92$ & 0.964 & $11.84 \pm 0.76$ & $12.14 \pm 1.08$ & 0.238 \\
\hline AFP, $n g / m L$ & $216.48 \pm 368.09$ & $316.31 \pm 447.99$ & 0.189 & $357.00 \pm 444.12$ & $367.45 \pm 474.58$ & 0.872 \\
\hline CA199, ng/mL & $26.33 \pm 20.32$ & $25.93 \pm 17.80$ & 0.878 & $23.65 \pm 21.76$ & $23.92 \pm 21.06$ & 0.763 \\
\hline Two & $70(88.6 \%)$ & $62(96.9 \%)$ & & $37(84.1 \%)$ & $74(88.1 \%)$ & \\
\hline Three & $9(11.4 \%)$ & $2(3.1 \%)$ & & $7(15.9 \%)$ & $10(11.9 \%)$ & \\
\hline
\end{tabular}

Table 1 (continued) 
Table 1 (continued)

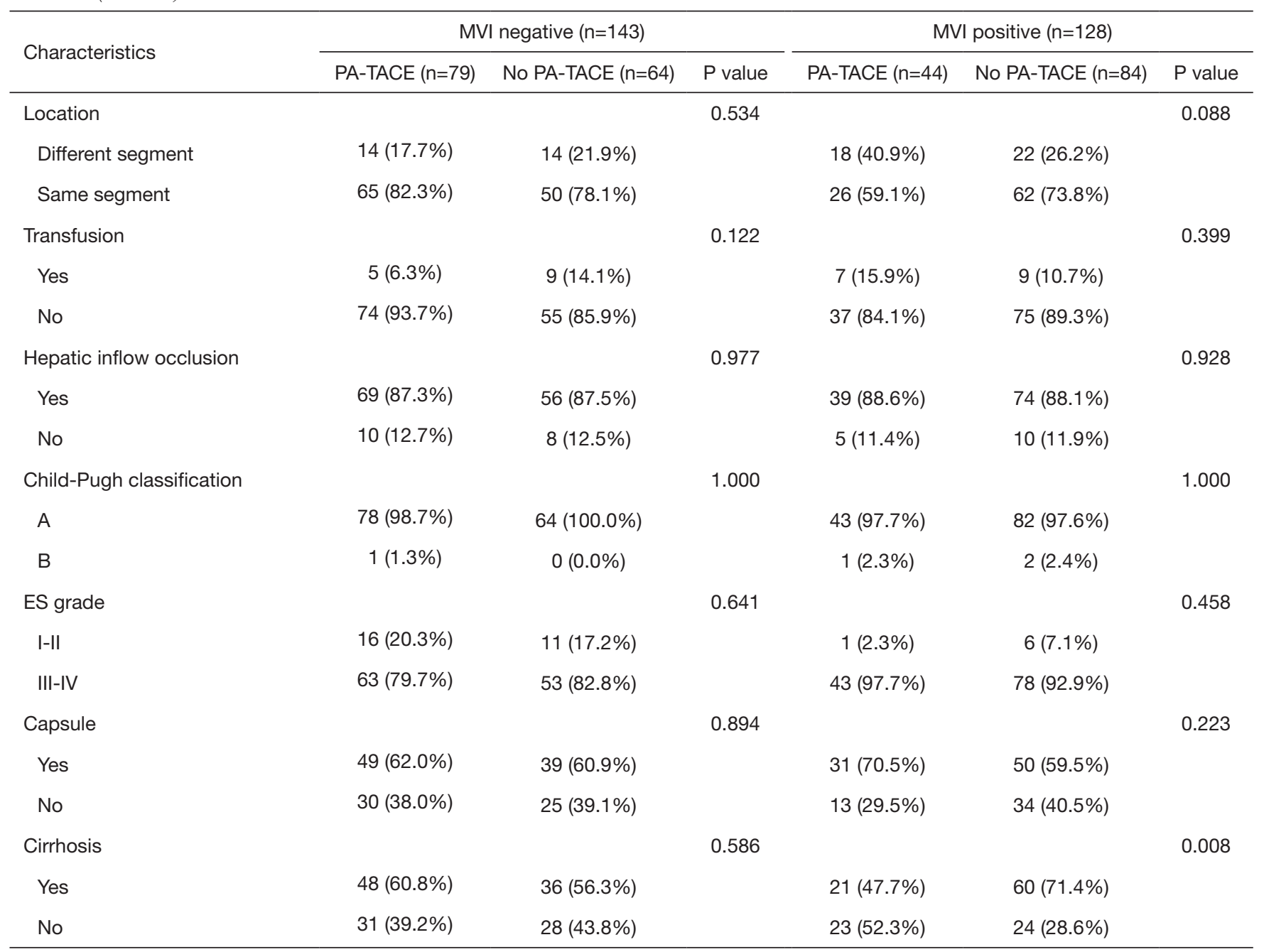

MVI, microvascular invasion; PA-TACE, postoperative adjuvant transarterial chemoembolization; TBIL, total bilirubin; TP, total protein; ALB, albumin; ALT, alanine transaminase; AST, aspartate aminotransferase; GGT, $\gamma$-glutamyl transpeptidase; ALP, alkaline phosphatase; WBC, white blood cells; RBC, red blood cells; PLT, platelets; INR, international normalized ratio; PT, prothrombin time; AFP, alpha fetal protein; CA199, carbohydrate antigen 19-9; ES, Edmondson-Steiner.

had nausea, vomiting fever, and pain after the PA-TACE, respectively.

\section{Prognostic factors for DFS and OS}

Results on univariate and multivariate analysis of the BCLC early stage MHCC patients for DFS and OS were shown in Table 2. Univariate analysis of the patients revealed male, higher $\gamma$-glutamyl transpeptidase (GGT), higher cancer antigen 19-9 (CA199), HBV DNA load $>10^{3}$ copy/mL, tumors located in different hepatic segment, EdmondsonSteiner grade III-IV, cirrhosis, MVI, and without PA-TACE were simultaneously risk factors for DFS (all $\mathrm{P}<0.05$ ). The independent risk factors of OS was higher GGT, higher alkaline phosphatase (ALP), lower white blood cell (WBC), lower platelet (PLT), larger overall diameter, three tumors, tumors located in different hepatic segment, cirrhosis, MVI, and without PA-TACE (all $\mathrm{P}<0.05$ ). Multivariate analysis showed that HBV DNA load $>10^{3}$ copy $/ \mathrm{mL}$ [hazard ratio (HR) 1.427; 95\% confidence interval (CI), 1.071-1.902; $\mathrm{P}=0.015]$, three tumors (HR 1.624; 95\% CI, 1.038-2.541; $\mathrm{P}=0.034$ ), MVI (HR 1.504; 95\% CI, 1.130-2.002; $\mathrm{P}=0.005$ ), and without PA-TACE (HR 1.433; 95\% CI, 1.070-1.918; $\mathrm{P}=0.016)$ were independent risk factors for poor DFS. 
Table 2 Uni- and multivariate analyses to identify predictors of disease-free survival and overall survival.

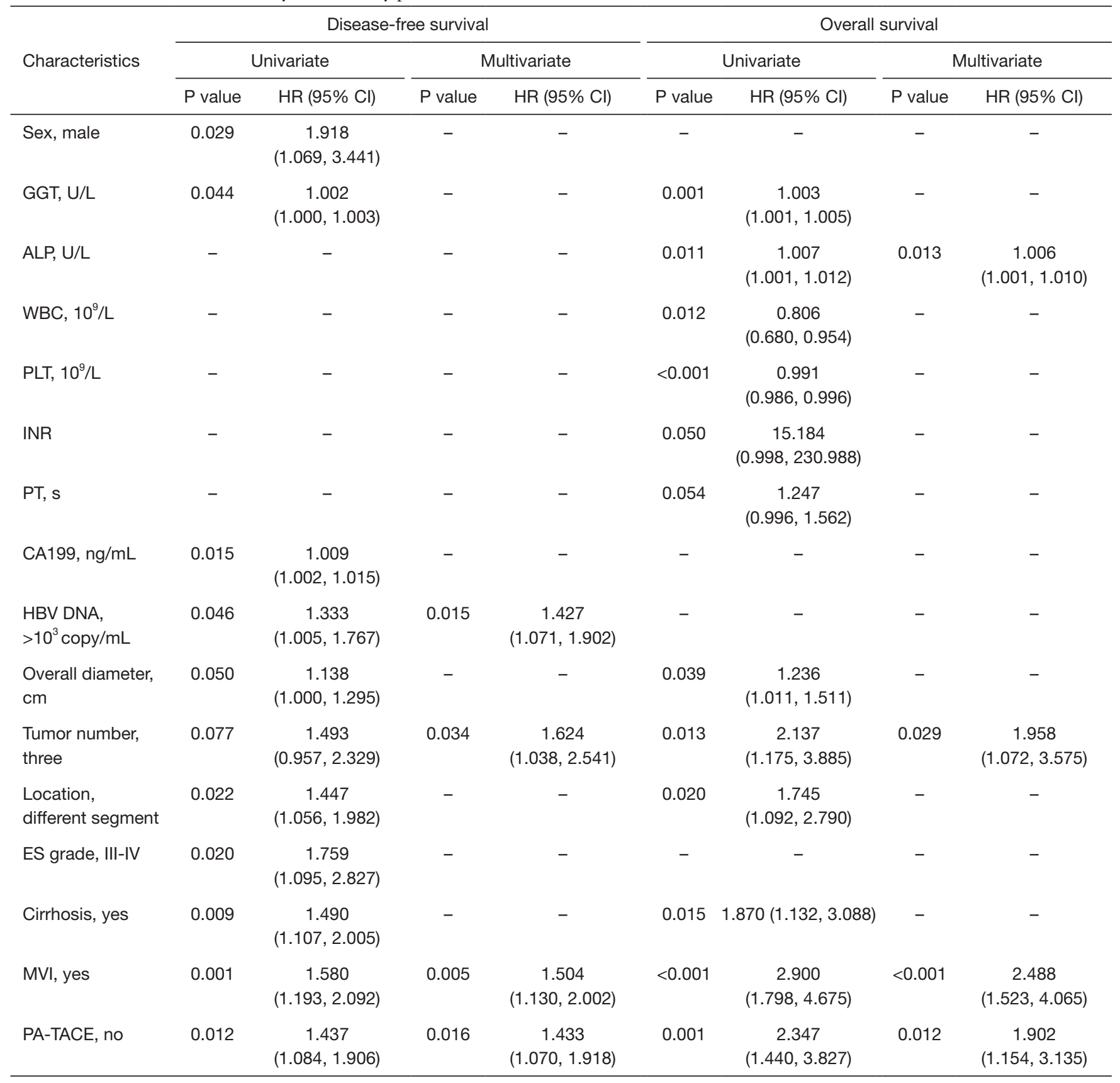

GGT, $\gamma$-glutamyl transpeptidase; ALP, alkaline phosphatase; WBC, white blood cells; PLT, platelets; INR, international normalized ratio; PT, prothrombin time; CA199, carbohydrate antigen 19-9; ES, Edmondson-Steiner; MVI, microvascular invasion; PA-TACE, postoperative adjuvant transarterial chemoembolization.

Higher ALP (HR 1.006; 95\% CI, 1.001-1.010; $\mathrm{P}=0.013$ ), three tumors (HR 1.958; 95\% CI, 1.072-3.575; $\mathrm{P}=0.029$ ), MVI (HR 2.488; 95\% CI, 1.523-4.065; $\mathrm{P}<0.001$ ), and without PA-TACE (HR 1.902; 95\% CI, 1.154-3.135; $\mathrm{P}=0.012$ ) were independent risk factors for poor OS.

\section{Efficacy of PA-TACE on the BCLC early stage MHCC patients with/without MVI}

Among patients with MVI, DFS was significantly better in patients treated by PA-TACE (median DFS, $22.23 v s$. 

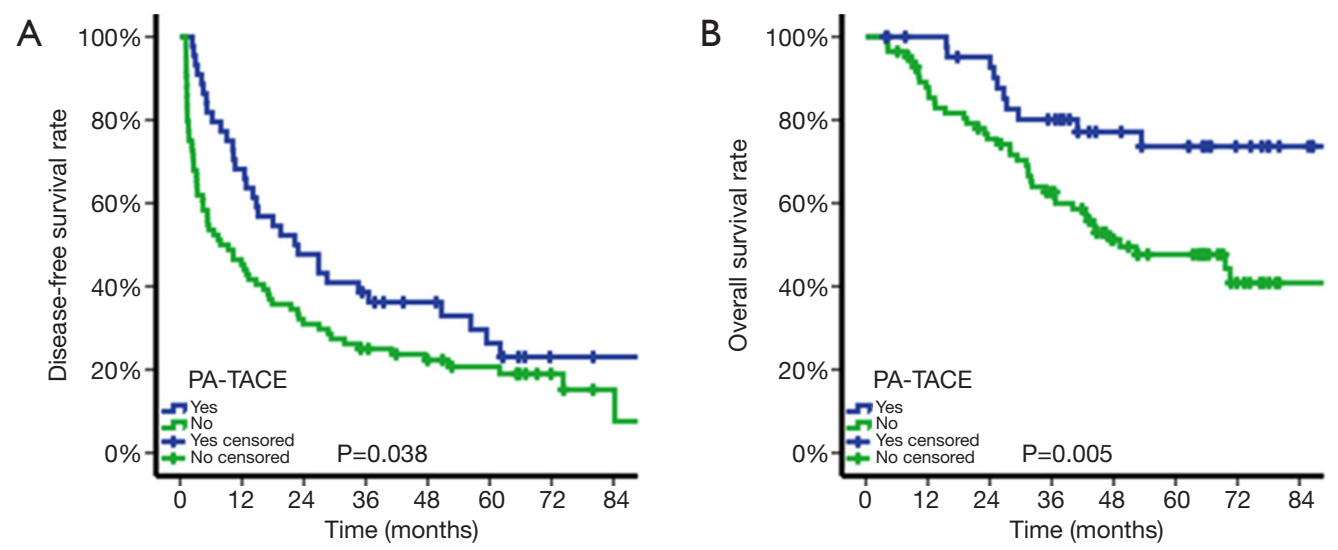

Figure 2 Cumulative incidence of disease-free survival (A) and overall survival (B) curves showing a comparison of the microvascular invasion positive patients with/without postoperative adjuvant transarterial chemoembolization. PA-TACE, postoperative adjuvant transcatheter arterial chemoembolization.
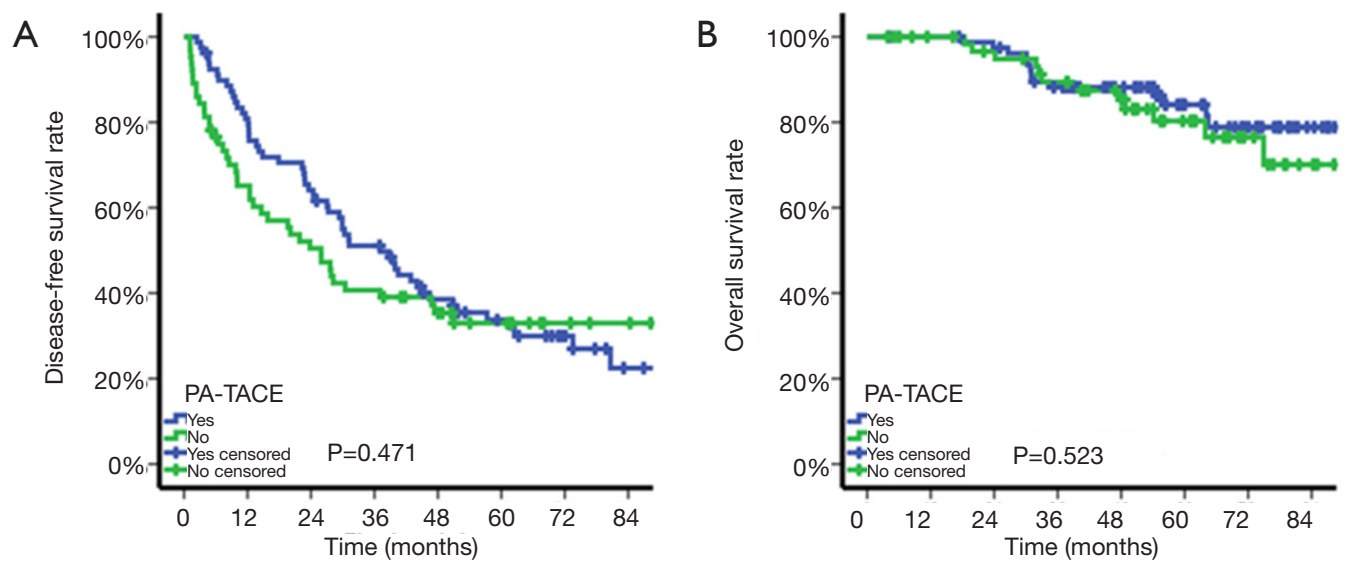

Figure 3 Cumulative incidence of disease-free survival (A) and overall survival (B) curves showing a comparison of the microvascular invasion negative patients with/without postoperative adjuvant transarterial chemoembolization. PA-TACE, postoperative adjuvant transcatheter arterial chemoembolization.

7.80 months; DFS at 1 year, $68.2 \%$ vs. $45.2 \%$; 3 years, $38.6 \%$ vs. $25.0 \%$; 5 years, $26.3 \%$ vs. $20.7 \%$; $\mathrm{P}=0.038$; Figure 2A). Similarly, OS was significantly improved in patients treated by PA-TACE than those treated by liver resection alone (OS at 1 year, $100.0 \%$ vs. $87.8 \% ; 3$ years, $80.1 \%$ vs. $62.7 \%$; 5 years, $73.6 \%$ vs. $47.7 \% ; \mathrm{P}=0.005$; Figure 2B). Among patients without MVI, DFS was similar between those with or without PA-TACE (median DFS, 37.13 vs. 25.90 months; DFS at 1 year, $80.8 \%$ vs. $65.1 \%$; 3 years, $51.1 \%$ vs. $40.7 \%$; 5 years, $33.7 \%$ vs. $33.0 \%$; $\mathrm{P}=0.471$; Figure $3 A$ ). Similar results were obtained for OS (OS at 1 year, $100.0 \%$ vs. 100.0\%; 3 years, $88.2 \%$ vs. $89.4 \%$; 5 years, $84.1 \%$ vs. $80.3 \%$; $\mathrm{P}=0.523$; Figure $3 B$ ).

\section{Impact of PA-TACE on the recurrence pattern and treatment strategy}

The recurrence pattern and treatment strategy of 196 relapsed patients was exhibited in Table 3. Although there was no significant difference of recurrence rate between the PA-TACE group and the no PA-TACE group, the PA-TACE group was less likely to recurrent in 2 years $(\mathrm{P}=0.001)$. Furthermore, compared with the PA-TACE group, the no PA-TACE group was more likely to select non-curative treatment methods, such as TACE, to control the recurrent tumor $(\mathrm{P}=0.005)$. The cumulative constituent ratio of early and late recurrence between patients with and 
Table 3 Recurrence pattern and treatment strategy

\begin{tabular}{|c|c|c|c|}
\hline Characteristics & PA-TACE & without PA-PACE & $P$ value \\
\hline Time & & & 0.001 \\
\hline$<2$ years (early recurrence) & $51(59.3 \%)$ & $89(80.9 \%)$ & \\
\hline$\geq 2$ years (late recurrence) & $35(40.7 \%)$ & $21(19.1 \%)$ & \\
\hline Intrahepatic & $83(96.5 \%)$ & $102(92.7 \%)$ & \\
\hline Extrahepatic & $1(1.2 \%)$ & $7(6.4 \%)$ & \\
\hline Intrahepatic + Extrahepatic & $2(2.3 \%)$ & $1(0.9 \%)$ & \\
\hline Intrahepatic tumor number & & & 0.378 \\
\hline Macrovascular invasion & & & 1.000 \\
\hline Yes & $3(3.5 \%)$ & $3(2.7 \%)$ & \\
\hline No & $83(96.5 \%)$ & $107(97.3 \%)$ & \\
\hline Treatment & & & 0.005 \\
\hline Re-resection & $14(16.3 \%)$ & $7(6.4 \%)$ & \\
\hline Ablation & $33(38.4 \%)$ & $24(21.8 \%)$ & \\
\hline TACE & $31(36.0 \%)$ & $63(57.3 \%)$ & \\
\hline Radiotherapy & $2(2.3 \%)$ & $5(4.5 \%)$ & \\
\hline
\end{tabular}

PA-TACE, postoperative adjuvant transarterial chemoembolization.

without PA-TACE was showed in Figure S1.

\section{Discussion}

With the advancement of hepatic surgery, hepatectomy has been considered as the first choice for the treatment of HCC (19-21). The stratified therapeutic options based on the BCLC staging system was the most widely used guidance in clinical practice $(22,23)$. Among all of HCC patients, those who met the BCLC early stage criteria could obtain good survival prognosis after curative treatment (24). Constrained by liver sources, many BCLC early stage MHCC patients selected hepatectomy instead of liver transplantation as the first treatment measure $(25,26)$. However, for MHCC, due to the increased number of tumor nodules and the presence of heterogeneity, the biological behaviors were more complex than those of solitary HCC $(27,28)$. Different clonal origin patterns of MHCC might prompt the distinguishing biological behaviors, postoperative recurrence rates, and long-term survival rates (29). The current view was that the IM type HCC stemmed from the hematogenous dissemination of primary tumor with a high degree of malignancy, while the MO type HCC was caused by poorly conserved liver background in which multiple hepatocytes undergone carcinogenesis simultaneously. Therefore, the IM type HCC has a higher postoperative recurrence rate and poorer survival prognosis than the MO type HCC (30).

MVI was defined as the presence of tumor emboli in a portal radicle vein, a vascular space lined by endothelial cells or large capsule vessel (31). The presence of MVI represented an aggressive biological behavior of the tumor and was one of the most critical factors predictive of intrahepatic metastasis (32). Kim et al. (11) detected that 
MVI was the most essential risk factors discriminating the IM type MHCC from the MO type MHCC $(\mathrm{P}<0.001)$. Li et al. (33) also demonstrated that MVI was the only independent risk factor for early intrahepatic recurrence ( $\mathrm{P}=0.006$; HR 2.397; 95\% CI, 1.290-4.451). Therefore, in view of the complex molecular pathological methods of clonal origin test and the lack of consensus on the criteria of the clonal origin, we believed that MVI could be an applied indication of the clonal origin pattern of MHCC.

The Cox proportional hazards regression models were conducted to identify that both the tumor itself (more tumor numbers and MVI) and the poor background liver (higher HBV DNA load and higher ALP) were correlated with short-term recurrence and survival which indicated the possibility of the IM type and the MO type original pattern in the BCLC early stage MHCC and their distinct prognostic implications for patients. Remarkably, MVI was associated with both poorer DFS (HR 1.504; $95 \%$ CI, 1.130-2.002; $\mathrm{P}=0.005)$ and OS (HR 2.488; 95\% CI, $1.523-4.065 ; \mathrm{P}<0.001)$ which prompted that the IM type MHCC has been accounted for part of these patients and the IM type MHCC might have a poorer clinical outcomes compared with the MO type MHCC. In addition, the three tumors was also an independent risk factor for poorer DFS (HR 1.624; 95\% CI, 1.038-2.541; $\mathrm{P}=0.034$ ) and OS (HR 1.958; 95\% CI, 1.072-3.575; $\mathrm{P}=0.029$ ) which meant that accompanied by the increased tumor number, the incidence of the IM type MHCC occurred more frequently. Considering MVI could not be obtained by preoperative parameters, effective intervention for the MVI positive BCLC early stage MHCC need further exploration.

TACE was recommended for the BCLC intermediate stage patients as the preferred treatment because of its specificity for killing hematogenous micrometastases and the accuracy of microcatheter superselection of tumor vessels $(27,34,35)$. However, whether TACE could be used as an adjuvant therapy of hepatectomy remained controversial. Zhou et al. (36) detected that preoperative TACE did not improve surgical outcome of the patients with resectable $\geq 5 \mathrm{~cm}$ HCC $(5$-year DFS, TACE $v s$. nonTACE, $8.9 \%$ vs. $12.8 \%, \mathrm{P}=0.372,5$-year OS, TACE $v$. non-TACE, $21.1 \%$ vs. $30.7 \%, \mathrm{P}=0.679$ ). Wang et al. (37) found that the HCC patients with intermediate (tumor size $>5 \mathrm{~cm}$ ) or high risk of recurrence (single tumor with MVI as well as 2 or 3 tumors) after curative liver resection could benefit from PA-TACE (3-year OS, TACE $v s$. nonTACE, $85.2 \%$ vs. $77.4 \%$; $\mathrm{P}=0.04)$. A meta-analysis showed that hepatectomy plus PA-TACE had superiority over hepatectomy alone in 1-year DFS (OR 1.86; 95\% CI, 1.382.51, $\mathrm{P}<0.001$ ), 3-year DFS (OR 2.37; 95\% CI, 1.73-3.24, $\mathrm{P}<0.001$ ), and 5-year DFS (OR 1.99; 95\% CI, 1.55-2.55, $\mathrm{P}<0.001$ ), as well as 1 -year OS (OR 2.16; 95\% CI, $1.75-$ 2.68, $\mathrm{P}<0.001$ ), 3-year OS (OR 1.77; 95\% CI, 1.48-2.13, $\mathrm{P}<0.001)$ and 5 -year OS (OR 1.92; 95\% CI, 1.44-2.56, $\mathrm{P}<0.001)$. However, subgroup analysis showed that only portal vein tumor thrombus, but not other factors related to recurrence risk (38). In conclusion, it was still essential to explore HCC patients suitable for PA-TACE.

According to our study, PA-TACE was an effective way to improve the prognosis of the BCLC early stage MHCC (DFS: HR 1.433; 95\% CI, 1.070-1.918; $\mathrm{P}=0.016$; OS: HR 1.902; 95\% CI, 1.154-3.135; $\mathrm{P}=0.012$ ). Interestingly, based on subgroup analysis, obvious prognostic improvement was only observed in the MVI positive group (DFS, $\mathrm{P}=0.038$; OS, $\mathrm{P}=0.005)$. Moreover, PA-TACE merely influenced on early recurrence instead of late recurrence $(\mathrm{P}=0.001)$. These results profiled that, for the BCLC early stage MHCC patients, postoperative pathological diagnosis should pay more attention to the judgment of MVI. For MVI positive MHCC patients, PA-TACE was a safe and necessary intervention to reduce postoperative recurrence and prolonged survival. For patients without MVI, PA-TACE was unnecessary. In other word, PA-TACE could effectively prevent recurrence of the IM type HCC rather than the MO type HCC. In addition, the detection rate of MVI was reaching $47.2 \%$ which represented that a large part of the BCLC early stage MHCC might be the IM type HCC. Therefore, it was not rigorous to classify MHCC patients into early or intermediate stage solely dependent on the diameter and number of tumor. The prediction of MVI in MHCC patients should be underlined in the reappraisal of the BCLC staging system.

The present study should be noted for several limitations. The drugs and dosages of PA-TACE could vary across medical centers. The standard of PA-TACE considering both the efficacy and safety should be formulated in future. Furthermore, objective molecular pathological techniques were still indispensable to distinguish the clonal origin of MHCC in clinical practice. Moreover, the present study was retrospective and involved patients from only one medical center, thereby highlighting the need for verification through large, multicenter, preferably prospective trials.

In conclusion, PA-TACE was a safe intervention and could effectively prevent tumor recurrence and improve the survival of the BCLC early stage MHCC patients with MVI. 


\section{Acknowledgements}

Funding: This work is supported by National Natural Science Foundation of China (grant No. 81472278) and Funds for Creative Research Groups of National Natural Science Foundation of China (grant No. 81521091).

\section{Footnote}

Conflicts of Interest: The authors have no conflicts of interest to declare.

Ethical Statement: The study protocol was approved by the Eastern Hepatobiliary Surgery Hospital Research Ethics Committee (No. EHBHKY2015-02-001).

\section{References}

1. Chen $\mathrm{W}$, Zheng R, Baade PD, et al. Cancer statistics in China, 2015. CA Cancer J Clin 2016;66:115-32.

2. Villanueva A, Llovet JM. Liver cancer in 2013: Mutational landscape of HCC--the end of the beginning. Nat Rev Clin Oncol 2014;11:73-4.

3. Llovet JM, Brú C, Bruix J. Prognosis of Hepatocellular Carcinoma: The BCLC Staging Classification. Semin Liver Dis 1999;19:329-38.

4. Llovet JM, Zucman-Rossi J, Pikarsky E, et al. Hepatocellular carcinoma. Nat Rev Dis Primers 2016;2:16018.

5. Wang JH, Wang CC, Hung CH, et al. Survival comparison between surgical resection and radiofrequency ablation for patients in BCLC very early/early stage hepatocellular carcinoma. J Hepatol 2012;56:412-8.

6. Kao WY, Chao Y, Chang CC, et al. Prognosis of Early-Stage Hepatocellular Carcinoma: The Clinical Implications of Substages of Barcelona Clinic Liver Cancer System Based on a Cohort of 1265 Patients. Medicine (Baltimore) 2015;94:e1929.

7. Li T, Wang SK, Zhi XT, et al. Cholecystectomy is associated with higher risk of early recurrence and poorer survival after curative resection for early stage hepatocellular carcinoma. Sci Rep 2016;6:28229.

8. Matsumoto Y, Fujii H, Matsuda M, et al. Multicentric occurrence of hepatocellular carcinoma: diagnosis and clinical significance. J Hepatobiliary Pancreat Surg 2001;8:435-40.

9. Wang B, Xia CY, Lau WY, et al. Determination of clonal origin of recurrent hepatocellular carcinoma for personalized therapy and outcomes evaluation: a new strategy for hepatic surgery. J Am Coll Surg 2013;217:1054-62.

10. Wang Z, Gong W, Shou D, et al. Clonal Origin of Hepatocellular Carcinoma and Recurrence After Liver Transplantation. Ann Transplant 2016;21:484-90.

11. Kim JM, Kwon CH, Joh JW, et al. Intrahepatic metastasis is more risky than multiple occurrence in hepatocellular carcinoma patients after curative liver resection. Hepatogastroenterology 2015;62:399-404.

12. Nishikawa H, Kita R, Kimura T, et al. Transcatheter arterial embolic therapies for hepatocellular carcinoma: a literature review. Anticancer Res 2014;34:6877-86.

13. Zhang Z, Liu Q, He J, et al. The effect of preoperative transcatheter hepatic arterial chemoembolization on disease-free survival after hepatectomy for hepatocellular carcinoma. Cancer 2000;89:2606-12.

14. Sun JJ, Wang K, Zhang CZ, et al. Postoperative Adjuvant Transcatheter Arterial Chemoembolization After R0 Hepatectomy Improves Outcomes of Patients Who have Hepatocellular Carcinoma with Microvascular Invasion. Ann Surg Oncol 2016;23:1344-51.

15. Dong ZR, Zhang PF, Wang CH, et al. Postoperative adjuvant transcatheter arterial chemoembolization for resectable multiple hepatocellular carcinoma beyond the Milan criteria: a retrospective analysis. Am J Cancer Res 2014;5:450-7.

16. Liu S, Guo L, Li H, et al. Postoperative Adjuvant Trans-Arterial Chemoembolization for Patients with Hepatocellular Carcinoma and Portal Vein Tumor Thrombus. Ann Surg Oncol 2018;25:2098-104.

17. Cong WM, Bu H, Chen J, et al. Practice guidelines for the pathological diagnosis of primary liver cancer: 2015 update. World J Gastroenterol 2016;22:9279-87.

18. Roayaie S, Blume IN, Thung SN, et al. A system of classifying microvascular invasion to predict outcome after resection in patients with hepatocellular carcinoma. Gastroenterology 2009;137:850-5.

19. Song TJ, Ip EW, Fong Y. Hepatocellular carcinoma: current surgical management. Gastroenterology 2004;127:S248-60.

20. Earl TM, Chapman WC. Hepatocellular carcinoma: resection versus transplantation. Semin Liver Dis 2013;33:282-92.

21. Dhir M, Melin AA, Douaiher J, et al. A Review and Update of Treatment Options and Controversies in the Management of Hepatocellular Carcinoma. Ann Surg 2016;263:1112-25. 
22. European Association For The Study Of The Liver; European Organisation For Research And Treatment Of Cancer. EASL-EORTC clinical practice guidelines: management of hepatocellular carcinoma. J Hepatol 2012;56:908-43. Erratum in: J Hepatol 2012;56:1430.

23. Heimbach JK, Kulik LM, Finn RS, et al. AASLD guidelines for the treatment of hepatocellular carcinoma. Hepatology 2018;67:358-80.

24. Forner A, Reig M, Bruix J. Hepatocellular carcinoma. Lancet 2018;391:1301-14.

25. Zhao WC, Yang N, Zhu N, et al. Patients with multiple hepatocellular carcinomas within the UCSF criteria have outcomes after curative resection similar to patients within the BCLC early-stage criteria. World J Surg 2012;36:1811-23.

26. Furukawa K, Shiba H, Horiuchi T, et al. Survival benefit of hepatic resection for hepatocellular carcinoma beyond the Barcelona Clinic Liver Cancer classification. J Hepatobiliary Pancreat Sci 2017;24:199-205.

27. Forner A, Gilabert M, Bruix J, et al. Treatment of intermediate-stage hepatocellular carcinoma. Nat Rev Clin Oncol 2014;11:525-35.

28. Nault JC, Galle PR, Marquardt JU. The role of molecular enrichment on future therapies in hepatocellular carcinoma. J Hepatol 2018;69:237-47.

29. Li SL, Su M, Peng T, et al. Clinicopathologic characteristics and prognoses for multicentric occurrence and intrahepatic metastasis in synchronous multinodular hepatocellular carcinoma patients. Asian Pac J Cancer Prev 2013;14:217-23.

30. Nomoto S, Hishida M, Inokawa Y, et al. Management of hepatocellular carcinoma should consider both tumor factors and background liver factors. Hepatobiliary Surg

Cite this article as: Wang H, Du PC, Wu MC, Cong WM. Postoperative adjuvant transarterial chemoembolization for multinodular hepatocellular carcinoma within the Barcelona Clinic Liver Cancer early stage and microvascular invasion. HepatoBiliary Surg Nutr 2018;7(6):418-428. doi: 10.21037/ hbsn.2018.09.05
Nutr 2014;3:82-5.

31. Feng LH, Dong H, Lau WY, et al. Novel microvascular invasion-based prognostic nomograms to predict survival outcomes in patients after R0 resection for hepatocellular carcinoma. J Cancer Res Clin Oncol 2017;143:293-303.

32. Rodriguez-Peralvarez M, Luong TV, Andreana L, et al. A systematic review of microvascular invasion in hepatocellular carcinoma: diagnostic and prognostic variability. Ann Surg Oncol 2013;20:325-39.

33. Li SH, Guo ZX, Xiao CZ, et al. Risk factors for early and late intrahepatic recurrence in patients with single hepatocellular carcinoma without macrovascular invasion after curative resection. Asian Pac J Cancer Prev 2013;14:4759-63.

34. Takayasu K, Arii S, Kudo M, et al. Superselective transarterial chemoembolization for hepatocellular carcinoma. Validation of treatment algorithm proposed by Japanese guidelines. J Hepatol 2012;56:886-92.

35. Takayasu K, Arii S, Ikai I, et al. Prospective cohort study of transarterial chemoembolization for unresectable hepatocellular carcinoma in 8510 patients. Gastroenterology 2006;131:461-9.

36. Zhou WP, Lai EC, Li AJ, et al. A prospective, randomized, controlled trial of preoperative transarterial chemoembolization for resectable large hepatocellular carcinoma. Ann Surg 2009;249:195-202.

37. Wang Z, Ren Z, Chen Y, et al. Adjuvant Transarterial Chemoembolization for HBV-Related Hepatocellular Carcinoma After Resection: A Randomized Controlled Study. Clin Cancer Res 2018;24:2074-81.

38. Zheng Z, Liang W, Wang D, et al. Adjuvant chemotherapy for patients with primary hepatocellular carcinoma: a meta-analysis. Int J Cancer 2015;136:E751-9. 

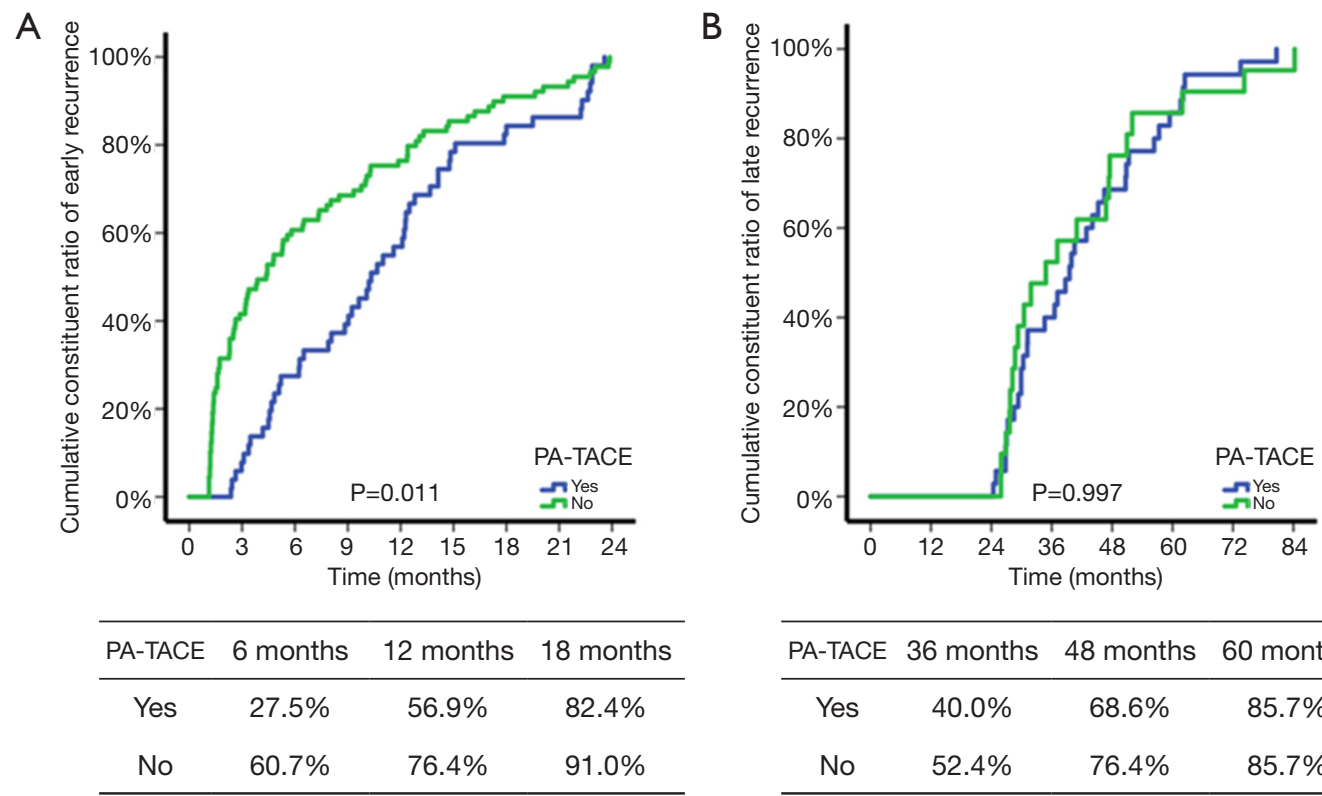

\begin{tabular}{cccc}
\hline PA-TACE & 36 months & 48 months & 60 months \\
\hline Yes & $40.0 \%$ & $68.6 \%$ & $85.7 \%$ \\
No & $52.4 \%$ & $76.4 \%$ & $85.7 \%$ \\
\hline
\end{tabular}

Figure S1 Cumulative constituent ratio of early (A) and late recurrence (B) showing a comparison of patients with/without postoperative adjuvant transarterial chemoembolization. PA-TACE, postoperative adjuvant transcatheter arterial chemoembolization. 